\title{
Risk of chemotherapy-induced pulmonary fibrosis is associated with polymorphic tumour necrosis factor-a2 gene
}

\author{
J. Libura*, F. Bettens", A. Radkowski", J.M. Tiercy\#, P.F. Piguet ${ }^{+}$
}

\begin{abstract}
Risk of chemotherapy-induced pulmonary fibrosis is associated with polymorphic tumour necrosis factor-a2 gene. J. Libura, F. Bettens, A. Radkowski, J.M. Tiercy, P.F. Piguet. (C) ERS Journals Ltd 2002.

ABSTRACT: In some patients, chemotherapy (CHT) of cancer can result in pulmonary inflammation and fibrosis, eventually leading to respiratory insufficiency.

As animal studies have underlined the importance of major histocompatibility complex (MHC) genes in the susceptibility to bleomycin (BLM)-induced pulmonary fibrosis, the authors typed human leukocyte antigen-DR (HLA-DR) and tumor necrosis factor (TNF) genes in patients treated for Hodgkin's disease by a therapy including bleomycin.

Patients were divided into pulmonary responders (PR) $(n=21)$ or nonresponders (PNR) $(n=20)$ on the basis of pulmonary alterations detected on chest radiography and the cumulated amount of BLM injected. The incidence of TNFa2, a microsatellite allele in the promoter region of the TNFB gene reported to be associated with increased TNF- $\alpha$ production, was significantly higher in PR than PNR (65\% versus 19\%). HLA-DRB1*15 showed a weak but nonsignificant association with the PR phenotype $(50 \%$ versus $14 \%)$, as well as HLA-DRB1 $03(30 \%$ versus $19 \%)$ and TNFA$308 * 2(30 \%$ versus $14 \%)$. TNFa2 and DR15 were independent risk factors and the occurrence of either genetic marker was $85 \%$ versus $29 \%$ in the PR and PNR groups respectively.
\end{abstract}

Thus, the polymorphic TNFa2 microsatellite is associated with a risk of chemotherapy-induced pulmonary fibrosis.

Eur Respir J 2002; 19: 912-918.

\begin{abstract}
*Postgraduate School of Molecular Medicine, Medical University, Warsaw and Dept of Tumour Pathology Institute of Oncology, Cracow, Poland. "Transplantation Immunology Unit, Hospital Cantonal, Geneva, Switzerland. Radiotherapy centre, Katowice, Poland. ${ }^{+}$Dept of Pathology, University of Geneva, Geneva, Switzerland.
\end{abstract}

Correspondence: P.F. Piguet, Dept of Pathology, 1 rue M. Servet, CMU, 1211 Geneva, Switzerland.

Fax: 41227025746

E-mail: pierre.piguet@medecine.unige.ch

Keywords: Bleomycin, Hodgkin's lymphoma, human leukocyte antigen-DR, pulmonary fibrosis, tumour necrosis factor

Received: April 202001

Accepted after revision October 172001

This work was supported in part by grants no. 31-56839.99, 31-57557.99, 32-52061.97 from the Swiss National Science Foundation. J. Libura is supported by the Marek-Rutkowski Foundation.
Various drugs are used for the chemotherapy (CHT) of cancer and the effectiveness of this treatment is limited by the susceptibility of the cancer cells and of the normal host tissues. In the latter case, damage to the lung can result in inflammation and fibrosis, sometimes with long delays after the administration of CHT [1]. Bleomycin (BLM), a chemotherapeuthic drug that belongs to a family of complex glycopeptides derived from Streptomyces verticillis with potent tumour killing properties, is known to induce a high incidence of pulmonary complications [2]. Toxicity of BLM for cancer cells is believed to be due to its capacity to react with deoxyribonucleic acid (DNA), producing chain scission. In addition, the pulmonary inflammation and fibrosis induced by BLM are aggravated by other interventions, such as oxygen administration or radiotherapy (RT). However, the incidence of pulmonary fibrosis (PF) concerns only a minority of patients treated with BLM, the reason for which is not yet known.

Administration of BLM in rodents is a classical model used to investigate the pathogenesis of PF [2]. In mice it has been reported that susceptibility to BLM-induced fibrosis varies to a great extent between strains. Some strains (e.g. CBA, C57BL6) are susceptible and others, notably the Balb/c, are nonresponders [3]. More refined analysis using mice with different major histocompatibility complexes (MHC) has shown that the nonresponder phenotype is linked to the H-2d (D) haplotype and, more precisely, to its telomeric part, which comprises the $\mathrm{D}$ and tumour necrosis factor (TNF) loci [4]. In addition, the importance of TNF in the development of PF has been demonstrated by showing that responder, but not nonresponder, mice increase their TNF production in the lung in response to BLM injection and that the severity of the fibrosis can be markedly attenuated by the administration of TNF antagonists [5, 6]. The immune response might also be important in the outcome of BLM-induced PF in mice since the disease is attenuated by T-lymphocyte depletion or in T-lymphocyte deprived mice [2, 5]. Furthermore, T-lymphocyte depletion abrogates the BLM-induced increase in TNF messenger ribonucleic acid in the lung [5]. These observations suggest that the MHC class II genes implicated in T-lymphocyte activation might also play a role in the initiation of the inflammatory cascade. 
In humans, the genes encoding TNF- $\alpha$ (TNFA) and the homologous but independently-regulated TNF- $\beta$ (TNFB) are located within the class III region of the MHC, between human leukocyte antigen (HLA)-B and HLA-DR [7]. The lymphotoxin- $\beta$ gene (LTB) lies 3 kilobases centrometric to TNFA and codes for a 33 kilodaltons glycoprotein. Lymphotoxin- $\beta$ forms a complex with TNF- $\beta$ on the surface of activated T, $\mathrm{B}$ and lymphokine-activated killer cells [8]. Numerous reports have documented polymorphisms within the TNF genes, which influence TNF production and disease susceptibility. HLA-DR3/DR4 subjects have been reported to produce higher TNF- $\alpha$ levels [9]. A single nucleotide polymorphism ( $\mathrm{G}$ to A transition) at position -308 in the promoter region of TNFA has been found to increase TNF- $\alpha$ transcription $6-8$-fold [10]. The TNFA-308*2 allele appears to be a risk factor for diseases such as asthma, severe malaria, diabetes, or coeliac disease [11-13]. Polymorphic microsatellites that flank the TNF genes have also proved to be informative markers of TNF- $\alpha$ production $[14,15]$. One of these, TNFa, located upstream of TNFB, has been reported to be associated with high (TNFa2) or low (TNFa6, a4) TNF- $\alpha$ production by blood monocytes [14]. TNFa2 has been reported to be a significant risk factor for insulin-dependent diabetes (IDD) or Leishmaniasis [15, 16].

$\mathrm{PF}$ is a complex group of diseases of multiple aetiologies. It is characterized by alveolar inflammation, with remodelling of the alveolar septa and an increase in the content of proteins of the extracellular matrix (ECM), notably collagen-1. As reviewed previously [17], studies of HLA phenotypes and some types of PF have not shown strong associations with HLA. The strongest association reported concerns HLA-DR 3 and the susceptibility to PF that is linked with systemic sclerosis [18]. Recently, a higher incidence of the TNFA-308*2 gene was reported in coal miners with pneumoconiosis [19]. There is presently no information regarding the possible association between CHT-induced PF and the HLA phenotype.

In the present study, the incidence of the HLADRB1, TNFa and TNFA-308 polymorphisms in groups of patients treated with $\mathrm{CHT}$, including BLM for Hodgkin's lymphoma (HL), were examined. The radiological response of the lung was graded and related to the total dose of BLM received, allowing classification of patients as pulmonary responders (PR) or pulmonary nonresponders (PNR). The authors report a significant association between the TNFa 2 microsatellite allele and susceptibility to CHTinduced PF.

\section{Methods}

\section{Patients}

Sixty-three patients ( 31 males) with HL were treated at the Institute of Oncology in Cracow, Poland, and were followed for $\geqslant 2.5 \mathrm{yrs}$, the median follow-up being 5.4 yrs. The disease was identified on the basis of lymph node biopsy and was staged according to the Ann Arbor system. The median age of patients was 40 yrs. Twelve patients were treated with RT alone and were not investigated further. Ten other patients were not included in the study for reasons including the presence of pulmonary abnormalities detected before treatment (1), an insufficient radiological record or a poor compliance with therapy (9). Forty-one patients with disease stage $>$ II, treated with repeated courses of hybrid CHT regimen containing mechlorethamine, vincristin, procarbazine, and prednisone/doxorubicin, BLM, and vinblastin as an induction therapy, as described by ConNors et al. [20], and for whom clinical and radiological data for $\geqslant 2.5 \mathrm{yrs}$ after the beginning of therapy were available, were investigated further for DNA studies. Three patients were treated with CHT alone. In 38 patients, CHT was followed by supradiaphragmatic RT of 39 Gray, in 26 fractions as consolidative treatment. CHT was administered within 8-40 weeks after diagnosis and the total dose of BLM within the CHT cocktail ranged between 15-509 mg. BLM dose intensity ranged between $1.5-10 \mathrm{mg} \cdot \mathrm{week}^{-1}$. Blood donors were used as control for the analysis of HLA and TNF polymorphisms.

\section{Score of pulmonary lesions}

The evaluation of the pulmonary response to CHT was based on chest radiography performed before and during the follow-up after therapy. The most recent radiographs, obtained $>2.5$ yrs after the beginning of therapy, were used for the lung score. Radiological abnormalities consistent with fibrosis, such as linear streaking, regional contraction, pleural thickening, tenting of the diaphragm, retraction of the hilum or mediastinum, lung scaring and patchy/discrete or solid consolidations, were recorded. Lesions were graded in accordance with the radiological criteria described in the Subjective, Objective, Medical and Analytical (SOMA) evaluation of injury score of the European Organization for Research and Treatment of Cancer (EORTC) [21], using a 0-4 scale defined as follows: 0 : normal lung; 1: discrete unilateral supradiaphragmatic densities; 2: diffuse and patchy densifications; 3: diffuse and patchy consolidations with area of confluence; and 4: diffuse patchy densification, involving scaring and contractions.

\section{Typing of human leukocyte antigen-DRB1 and tumour necrosis factor genes}

DNA was extracted from frozen peripheral blood samples using standard proteinase $\mathrm{K}$ and phenolchloroform procedure. HLA-DRB1 typing was performed by polymerase chain reaction sequence-specific oligonucleotide probes (PCR-SSOP) hybridization [22].

TNFa microsatellite polymorphism was determined by using the PCR primers IR2 5'-GCCTCTAGATTTCATCCAGCCACA and flourescein fluorescencelabelled IR4 5' CCTCTCTCCCCTGCAACACACA [14]. PCR reactions contained $10 \mathrm{pmol}$ of each primer, $0.4 \mu \mathrm{g}$ DNA in $1.75 \mathrm{mM} \mathrm{MgCl}_{2}, 10 \mathrm{mM}$ Tris (pH 8.8), $50 \mathrm{mM} \mathrm{KCl}$ buffer, $200 \mu \mathrm{M}$ deoxyribonucleotide triphosphates, $10 \mu \mathrm{g} \cdot \mathrm{mL}^{-1}$ bovine serum 
albumin (BSA), and 0.5 units Taq polymerase (Invitrogen BV, Leek, the Netherlands). Cycling conditions were as follows: denaturation for $3 \mathrm{~min}$ at $94^{\circ} \mathrm{C}$, followed by 24 cycles of $30 \mathrm{~s}$ at $94^{\circ} \mathrm{C} / 30 \mathrm{~s}$ at $54^{\circ} \mathrm{C}$ (decreasing every cycle by $0.2^{\circ} \mathrm{C}$ ) $/ 30 \mathrm{~s}$ at $72^{\circ} \mathrm{C}$ in a Techne Progene termocycler (Applied Biosystems, Rotkreuz, Switzerland). The PCR $(1 \mu \mathrm{L})$ product was mixed with formamide and $0.4 \mu \mathrm{L}$ of internal size standard (TAMRA; Applied Biosystems), heat denatured and analysed by electrophoresis on an ABI 310 (PE Biosystem) capillary DNA sequencer using the Genescan program. The sizes of the PCR products were assigned based on TNFa alleles determined from control cell lines of known genotype: QBL (93 base pairs (bp)), AMALA (95 bp), WT51 (99 bp), YAR (111 bp), HHK (113 bp), corresponding to the TNFa1, TNFa2, TNFa4, TNFa10 and TNFa11 alleles, respectively [14].

TNF-308 diallelic polymorphism (guanine (G)/ adenine (A)) was determined as described previously [23]. Briefly, the TNF-308*1 allele ( $\mathrm{G}$ at position -308) was determined using primer pair $\mathrm{C} 1 / \mathrm{C} 2$ and the TNF-308*2 allele (A at position -308) with the primer pair $\mathrm{C} 1 / \mathrm{C} 3$. Primers were as follows. $\mathrm{C} 1$ : 5'-TCTCGGTTTCTTCTCCATCG; C2: 5'-ATAGGTTTTGAGGGGCATGG; and C3: 5'-ATAGGTTTTGAGGGGCATGA. HLA-DRB primers were included in each reaction as internal PCR controls. Each PCR contained 10 pmol of each of the primers $\mathrm{C} 1 / \mathrm{C} 2$ or $\mathrm{C} 1 / \mathrm{C} 3,3$ pmols of the HLA-DR primers DRBP1/DRBP2 [22], $200 \mathrm{ng}$ DNA in $1.75 \mathrm{mM}$ $\mathrm{MgCl}_{2}, 10 \mathrm{mM}$ Tris-HCl (pH 8.8), $50 \mathrm{mM} \mathrm{KCl}$ buffer, $200 \mu \mathrm{M}$ deoxyribonucleotide triphosphates (dNTPs), $10 \mu \mathrm{g} \cdot \mathrm{mL}^{-1}$ BSA and 0.5 units of Taq polymerase. The reaction mixture was denatured for $3 \mathrm{~min}$ at $94^{\circ} \mathrm{C}$, followed by 10 cycles of $30 \mathrm{~s}$ at $95^{\circ} \mathrm{C} / 45 \mathrm{~s}$ at $65^{\circ} \mathrm{C} / 40 \mathrm{~s}$ at $72^{\circ} \mathrm{C}$ and 19 cycles of $30 \mathrm{~s}$ at $94^{\circ} \mathrm{C} / 75 \mathrm{~s}$ at $63^{\circ} \mathrm{C} / 45 \mathrm{~s}$ at $72^{\circ} \mathrm{C}$. PCR products were electrophoresed on a ethidium bromide containing $1.5 \%$ agarose gel and visualized under ultraviolet light.

\section{Statistical evaluation}

Phenotype frequency was calculated from the number of patients or controls possessing the phenotype divided by the total number of subjects in the sample. Gene frequency was calculated using the Arlequin computer package (Arlequin Version 2.0; Genetics and Biometry Lab, Dept of Anthropology, University of Geneva, Geneva, Switzerland). The association of HLA-DR and TNF alleles within groups of patients was tested using the Chi-squared test. p-Values were corrected $(\mathrm{Pc})$ using the Bonferroni correction applied to all significant differences.

\section{Results}

Human leukocyte antigen-DRB1 and tumour necrosis factor polymorphisms in Hodgkin's lymphoma

In order to isolate the effects of CHT on the lung from the effects of the basic disease and to discriminate between the effects of TNF polymorphisms and those of other HLA-DR genes, DNA in all patients and controls was typed for the TNFa, TNF308 and HLA-DRB1 polymorphisms [24]. The HLADRB1 phenotypic frequencies in $41 \mathrm{HL}$ patients and 51 healthy controls are shown in table 1 . A somewhat higher frequency of HLA-DRB $1 * 15$ and HLADRB1*11 was detected in HL patients as compared to normal subjects, whereas DRB1*07 and DRB1*13 were less frequent in the HL patients (table 1). These associations, although nonsignificant, were in accordance with those reported in a study on patients with nodular sclerosing HL [25].

With regard to the TNF polymorphic genes, no significant difference in the distribution of the TNFA308* alleles was observed between healthy controls and HL patients (table 1), in accordance with a previous study of $36 \mathrm{HL}$ patients [26]. The overall frequency distribution of the TNFa microsatellites in controls was also similar to that reported previously in a normal population [27] and was not different from that observed in HL patients (table 1).

\section{Response of the lung to chemotherapy}

As shown on figure 1, the cumulative incidence of pulmonary abnormalities of different grades increased up to $\sim 4$ yrs after the beginning of CHT

Table 1.-Human leukocyte antigen-DR and tumour necrosis factor (TNF)a in controls and in Hodgkin lymphoma patients

$$
\frac{\text { Controls }}{n \quad \%} \frac{\text { Hodgkin }}{n \quad \%} \begin{gathered}
\text { Chi- } \\
\text { squared }
\end{gathered} \text { p-value } \mathrm{Pc}
$$

\begin{tabular}{|c|c|c|c|c|c|c|c|}
\hline Subjects & 51 & & 41 & & & & \\
\hline DRB $1 * 01$ & 11 & 21.6 & 10 & 24.4 & 0.1027 & 0.748 & \\
\hline $\mathrm{DRB} 1 * 15$ & 11 & 21.6 & 13 & 31.7 & 1.2116 & 0.271 & \\
\hline DRB $1 * 16$ & 3 & 5.9 & 7 & 17.0 & 1.8964 & 0.168 & \\
\hline DRB $1 * 03$ & 11 & 21.6 & 10 & 24.4 & 0.1027 & 0.748 & \\
\hline DRB $1 * 04$ & 15 & 29.4 & 8 & 19.5 & 1.1879 & 0.275 & \\
\hline $\mathrm{DRB} 1 * 11$ & 12 & 23.5 & 15 & 36.6 & 1.8684 & 0.171 & \\
\hline $\mathrm{DRB} 1 * 12$ & 2 & 3.9 & 1 & 2.4 & 0.0370 & 0.847 & \\
\hline $\mathrm{DRB} 1 * 13$ & 13 & 25.5 & 4 & 9.8 & 37352 & 0.053 & \\
\hline $\mathrm{DRB} 1 * 14$ & 3 & 5.9 & 2 & 4.9 & 0.0632 & 0.801 & \\
\hline DRB $1 * 07$ & 14 & 27.5 & 4 & 9.8 & 4.5222 & 0.033 & 0.43 \\
\hline DRB $1 * 08$ & 4 & 7.8 & 0 & 0 & 1.7404 & 0.187 & \\
\hline DRB $1 * 09$ & 0 & & 0 & 0 & & & \\
\hline DRB $1 * 10$ & 0 & & 2 & 4.9 & 0.7665 & 0.381 & \\
\hline TNFa2 & 17 & 33.3 & 17 & 41.4 & 0.6447 & 0.421 & \\
\hline TNFa3 & 1 & 1.9 & 2 & 4.9 & 0.0370 & 0.847 & \\
\hline TNFa4 & 7 & 13.7 & 9 & 21.9 & 1.0704 & 0.300 & \\
\hline TNFa5 & 4 & 7.8 & 8 & 19.5 & 2.7286 & 0.098 & \\
\hline TNFa6 & 11 & 21.5 & 12 & 29.3 & 0.7186 & 0.396 & \\
\hline TNFa7 & 6 & 11.7 & 11 & 26.8 & 3.4240 & 0.064 & \\
\hline TNFa8 & 1 & 1.9 & 2 & 4.9 & 0.0370 & 0.847 & \\
\hline TNFa9 & 6 & 11.7 & 4 & 9.7 & 0.0008 & 0.976 & \\
\hline TNFa10 & 13 & 25.5 & 18 & 43.9 & 3.4487 & 0.063 & \\
\hline TNFa11 & 11 & 21.5 & 9 & 21.9 & 0.0019 & 0.964 & \\
\hline TNFa12 & 1 & 1.9 & 3 & 7.3 & 0.5444 & 0.460 & \\
\hline TNF-308*1 & 50 & 98.0 & 40 & 97.6 & 0.3167 & 0.573 & \\
\hline TNF-308*2 & 11 & 21.6 & 9 & 21.9 & 0.0019 & 0.964 & \\
\hline
\end{tabular}

Pc: Bonferroni correction. 


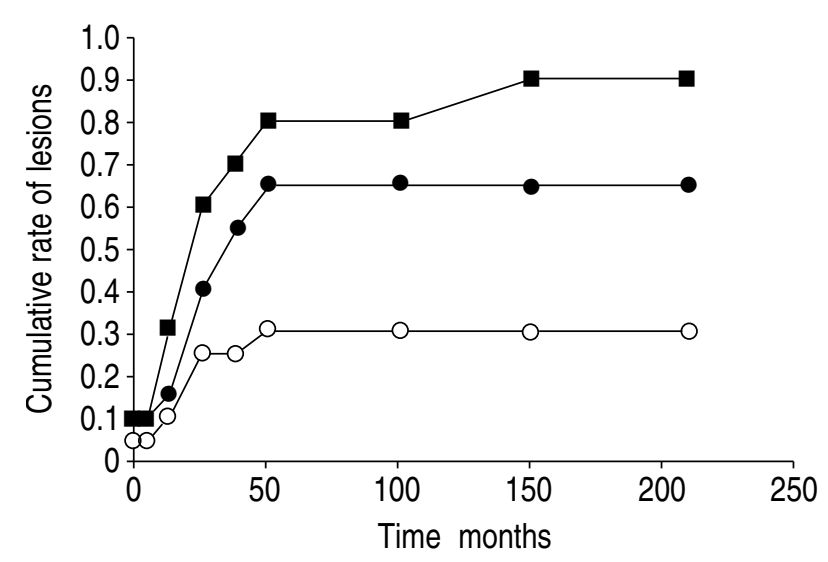

Fig. 1.-Cumulative incidence of pulmonary lesions in patients as a function of time after the initiation of chemotherapy (CHT) $(n=3)$ or CHT associated with radiotherapy $(n=38)$. Lung lesions scored $<2,(\boldsymbol{\square}),<3(\bullet)$ or $<4(\bigcirc)$.

and remained relatively stable thereafter. To evaluate responsiveness to $\mathrm{CHT}$, the most recent radiographs, obtained $>2.5$ yrs after the beginning of $\mathrm{CHT}$, were used. When the lung score was examined in relation to the total dose of BLM injected, no significant correlation was evident (fig. 2).

To evaluate the HLA-DRB1 and TNF genotypes of patients with CHT-induced pulmonary complications, a total of 41 patients treated with CHT alone $(n=3)$ or CHT associated with RT $(n=38)$ for Hodgkin's stage $>$ II were analysed. Twenty-one of these patients had enlarged mediastinal lymph node without radiological involvement of the lung parenchyma. In order to score the response of the lung to CHT, an index of responsiveness, which took into account the lung score determined 4 yrs after the beginning of the CHT, was divided by the total dose of BLM injected in $\mathrm{mg}\left(1 \times 10^{2}\right)$, based upon the assumption that the pulmonary response is directly related to the severity of the lung lesions and indirectly related with the total dose of BLM. On the basis of this index, patients were ranked in decreasing order of responsiveness (table 2). Patients with a lung score $>2.5$ were classified as

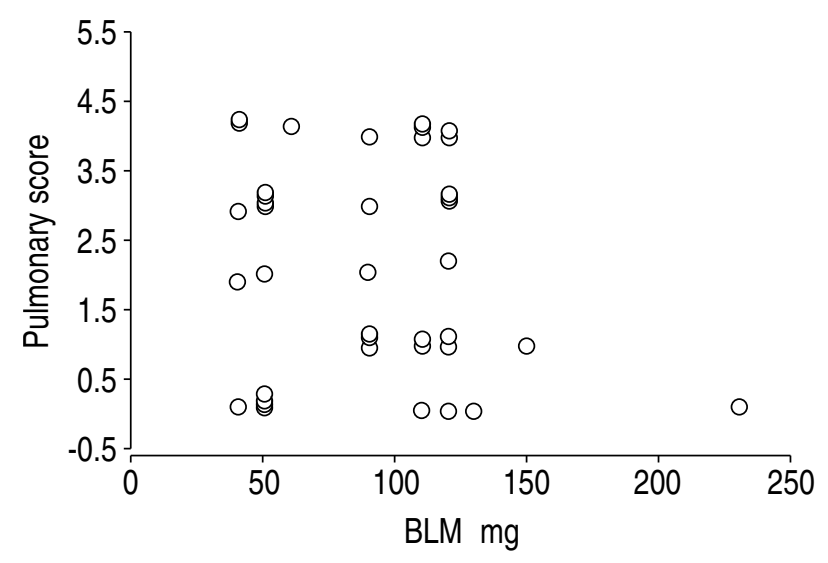

Fig. 2.-Patient pulmonary score in relation to the total dose of bleomycin (BLM) used in chemotherapy. Coeffiecient of determination $<0.03$.
PR, while those with a score $<2.5$ were classified as PNR. The mean \pm SD ages in both groups were $40 \pm 12$ and $35 \pm 15$ for PR and PNR, respectively. The male/ female ratios were $12 / 20$ and $7 / 21$ in PR and PNR, respectively. Histological types were similar in both groups, with a mixed population of cell types (7 of 15 and 8 of 15 in PR and PNR, respectively). Involvement of the mediastinal LN was present in 11 of 21 of the PNR and 10 of 20 of the PR, i.e. it was not associated with the occurrence of parenchymal alterations.

Human leukocyte antigen-DRBI and tumour necrosis factor gene polymorphisms in the pulmonary response to chemotherapy

Comparison of the TNFa polymorphisms in the PR and PNR showed a significant increase of TNFa2 in PR (tables 2 and 3). In contrast, the frequency of $\mathrm{TNFa} 2$ was similar in normal subjects and in $\mathrm{HL}$ patients (table 1). The TNFa6 allele was slightly more frequent and the TNFa4 allele slightly less frequent in PNR individuals. Other polymorphic genes reported to be associated with increased TNF$\alpha$ production are HLA-DR3 [28] and TNFA-308*2, a diallelic polymorphism located in the promoter of the TNFA gene [9]. These two alleles were increased, although not significantly, in PR patients (table 3). A slight increase of the DRB $1 * 15$ genotype in the PR compared to the PNR group (50\% versus $14 \%$, $\mathrm{Pc}=0.18$ ) (table 3) was also observed.

A calculation of linkage disequilibrium between the HLA-DRB1 gene and the TNFa loci did not show any significant linkage, neither in the total population of patients and controls $(\mathrm{p}=0.332)$ nor in patients $(p=0.897)$ or controls alone $(p=0.257)$. The combined analysis of $\mathrm{DRB} 1 * 15$ and $\mathrm{TNFa} 2$ polymorphisms (table 4) showed that a similar percentage of TNFa2positive patients was found in the DRB $1 * 15$-positive $(60 \%)$ and DRB1*15-negative $(70 \%)$ PR, whereas this percentage was lower but not markedly different in the DRB1*15-positive $(33.3 \%)$ and DRB1*15negative $(16.6 \%)$ PNR. In controls, the DRB1*15positive and DRB1*15-negative subjects contained similar proportions of $\mathrm{TNFa} 2,27.3 \%$ and $32.5 \%$, respectively. These figures argue against a linkage between DRB1*15 and TNFa2 and suggest that $\mathrm{DRB} 1 * 15$ and $\mathrm{TNFa} 2$ are independent risk factors in BLM-induced fibrosis. In contrast, DRB1*03 and TNF-308*2 exhibited a highly significant linkage disequilibrium $(p<0.0001)$. A linkage of DRB1*03 with $\mathrm{TNFa} 2$ has been previously reported and was also evident in the HL patients in this study, whereas DRB1*15 was found to be associated with TNFa11 in the A3-B7-DR2 haplotype [27].

\section{Discussion}

The poor or absent correlation between BLM dose and pulmonary response suggests that the genetic constitution of the host is of importance in the occurrence of pulmonary complications, an interpretation 
Table 2. - Pulmonary response to chemotherapy (CHT), human leukocyte antigen (HLA) and tumour necrosis factor (TNF) genes

\begin{tabular}{|c|c|c|c|c|c|c|c|}
\hline \multirow[t]{2}{*}{ Patient no. } & \multicolumn{2}{|c|}{ BLM dose mg } & \multirow[t]{2}{*}{ Lung score } & \multirow{2}{*}{$\begin{array}{l}\text { Lung responder } \\
\text { score }\end{array}$} & \multirow[t]{2}{*}{ HLA-DRB $1 *$} & \multirow[t]{2}{*}{ TNF-308 } & \multirow[t]{2}{*}{$\mathrm{TNFa}$} \\
\hline & Total & Per week & & & & & \\
\hline 1 & 30 & 4.2 & 4 & 13.3 & $15 / 11$ & $1 / 1$ & $2 / 11$ \\
\hline $2^{+}$ & 30 & 10.0 & 4 & 13.3 & $15 / x$ & $1 / 1$ & $2 / 6$ \\
\hline 3 & 30 & 8.8 & 3 & 10.0 & $04 / 11$ & $1 / 1$ & $2 / 9$ \\
\hline 4 & 60 & 6.0 & 4 & 6.6 & $03 / 01$ & $1 / 2$ & $2 / 11$ \\
\hline 5 & 45 & 5.2 & 3 & 6.6 & $03 / 11$ & $1 / 2$ & $2 / 2$ \\
\hline 6 & 45 & 5.2 & 3 & 6.6 & $04 / 11$ & $1 / 1$ & $6 / 11$ \\
\hline 7 & 45 & 11.0 & 3 & 6.6 & $01 / 04$ & $1 / 1$ & $6 / 10$ \\
\hline 8 & 45 & 5.2 & 3 & 6.6 & $15 / 03$ & $1 / 1$ & $2 / 9$ \\
\hline 9 & 45 & 10.0 & 2 & 4.4 & $15 / 01$ & $1 / 1$ & $6 / 11$ \\
\hline 10 & 90 & 4.0 & 4 & 4.4 & $15 / 11$ & $1 / 1$ & $7 / 10$ \\
\hline 11 & 90 & 4.5 & 4 & 4.4 & $15 / x$ & $1 / 2$ & $2 / 11$ \\
\hline 12 & 105 & 2.7 & 4 & 3.8 & $03 / 13$ & $1 / 1$ & $2 / 10$ \\
\hline 13 & 110 & 5.2 & 4 & 3.8 & $16 / 03$ & $1 / 2$ & $2 / 10$ \\
\hline 14 & 120 & 6.0 & 4 & 3.3 & $15 / 04$ & $1 / 1$ & $2 / 10$ \\
\hline 15 & 105 & 1.9 & 4 & 3.8 & 11/x & $1 / 1$ & $4 / 5$ \\
\hline 16 & 30 & 6.0 & 1 & 3.3 & $11 / 01$ & $1 / 1$ & $2 / 6$ \\
\hline 17 & 110 & 6.0 & 4 & 3.3 & $01 / 15$ & $1 / 1$ & $5 / 9$ \\
\hline 18 & 90 & 9.0 & 3 & 3.3 & $01 / 15$ & $1 / 1$ & $6 / 10$ \\
\hline 19 & 105 & 12.2 & 3 & 2.8 & $03 / 16$ & $1 / 2$ & $2 / 6$ \\
\hline 20 & 120 & 2.8 & 3 & 2.5 & $15 / 07$ & $1 / 2$ & $2 / 6$ \\
\hline $21^{+}$ & 210 & 5.0 & 4 & 1.9 & $03 / 11$ & $1 / 1$ & $2 / 4$ \\
\hline 22 & 120 & 15.0 & 2 & 1.6 & $16 / 01$ & $1 / 2$ & $4 / 11$ \\
\hline 23 & 90 & 4.5 & 1 & 1.1 & $07 / 11$ & $1 / 1$ & $7 / 10$ \\
\hline 24 & 105 & 13.0 & 1 & 1.0 & $03 / 10$ & $1 / 1$ & $9 / 11$ \\
\hline 25 & 105 & 5.0 & 1 & 1.0 & $04 / 13$ & $1 / 1$ & $2 / 6$ \\
\hline 26 & 90 & 4.2 & 1 & 1.1 & $03 / 04$ & $1 / 1$ & $10 / 11$ \\
\hline 27 & 120 & 4.0 & 1 & $<1$ & $04 / 07$ & $1 / 1$ & $7 / 14$ \\
\hline $28^{+}$ & 120 & 4.2 & 1 & $<1$ & $13 / 04$ & $1 / 1$ & $4 / 7$ \\
\hline 29 & 150 & 4.3 & 1 & $<1$ & $01 / x$ & $1 / 2$ & $5 / 7$ \\
\hline 30 & 135 & 7.6 & 0 & $<1$ & $15 / \mathrm{x}$ & $1 / 1$ & $4 / 10$ \\
\hline 31 & 45 & 6.3 & 0 & $<1$ & $16 / 11$ & $1 / 1$ & $5 / 6$ \\
\hline 32 & 105 & 4.1 & 0 & $<1$ & $12 / 13$ & $1 / 1$ & $11 / 12$ \\
\hline 33 & 90 & 5.8 & 0 & $<1$ & $03 / 16$ & $2 / 2$ & $2 / 4$ \\
\hline 34 & 30 & 2.9 & 0 & $<1$ & $11 / \mathrm{x}$ & $1 / 1$ & $10 / 10$ \\
\hline 35 & 30 & 4.0 & 0 & $<1$ & $01 / 11$ & $1 / 1$ & $6 / 9$ \\
\hline 36 & 45 & 5.0 & 0 & $<1$ & $16 / 11$ & $1 / 1$ & $3 / 10$ \\
\hline 37 & 45 & 5.2 & 0 & $<1$ & $11 / 14$ & $1 / 1$ & $10 / 11$ \\
\hline 38 & 509 & 8.7 & 4 & $<1$ & $16 / 11$ & $1 / 1$ & $10 / 10$ \\
\hline 39 & 90 & 4.5 & 0 & $<1$ & $15 / 04$ & $1 / 1$ & $2 / 8$ \\
\hline 40 & 120 & 5.7 & 0 & $<1$ & $15 / 10$ & $1 / 1$ & $9 / 11$ \\
\hline 41 & 225 & 8.0 & 0 & $<1$ & $01 / 07$ & $1 / 1$ & $4 / 7$ \\
\hline
\end{tabular}

BLM: Bleomycin. ${ }^{\#}$ : patients were treated with CHT associated with radiotherapy; ${ }^{\uparrow}$ : patients were ranked according to the lung responder score; ${ }^{+}$: patients were treated with $\mathrm{CHT}$ alone.

in accordance with the observations made in mice [4]. The present results indicate that $\mathrm{TNFa} 2$, a polymorphic TNF allele, is a significant risk factor.

The monocytes from TNFa2 carriers have been reported to produce higher TNF- $\alpha$ levels in response to lipopolysaccharide in vitro, although surprisingly this allele did not influence the production of TNF- $\beta$ [15]. However, in a study of 22 heart transplant patients, no association between the serum TNF- $\alpha$ level and the TNFa2 allele was observed [29]. These discrepancies might be due to the fact that the serum level, which results from a balance between synthesis and clearance, does not reflect synthesis as closely as the culture supernatant from activated leukocytes. Since macrophages are not the exclusive source of TNF during CHT-induced PF [5], the correlation between these polymorphisms and TNF production by other cells remains to be explored. The TNFa2 allele has been reported to be associated with diseases such as IDD [15] and cutaneous Leishmaniasis [16]. DRB1*15 was also weakly associated with risk of pulmonary complication. This finding is intriguing because, so far, this allele has not been reported to be associated with immune or inflammatory diseases. DRB1*15 and $\mathrm{TNFa} 2$ were not genetically linked and, accordingly, when the presence of either DRB $1 * 15$ or TNFa 2 was considered, a highly significant increase in the occurrence of either genetic marker in PR as compared to PNR was evident (85\% versus $28.6 \%, \mathrm{Pc}=0.0068)$ (table 3 ).

In conclusion, the association of the risk of chemotherapy-induced pulmonary complication with 
Table 3.-Tumour necrosis factor (TNF)a and human leukocyte antigen (HLA)-DR polymorphic genes in pulmonary responders to chemotherapy

$$
\frac{\mathrm{PR}}{\mathrm{n} \%} \frac{\mathrm{PNR}}{\mathrm{n} \quad \%} \begin{gathered}
\text { Chi- } \\
\text { squared }
\end{gathered} \text {-value } \mathrm{Pc}
$$

\begin{tabular}{lccccccc}
\hline $\begin{array}{l}\text { Subject n } \\
\text { TNF }\end{array}$ & 20 & & 21 & & & & \\
a2 & 13 & 65 & 4 & 19 & 8.9122 & 0.0028 & 0.033 \\
a3 & 0 & 0 & 1 & 5 & 0.0006 & 0.9803 & \\
a4 & 1 & 5 & 6 & 29 & 2.5275 & 0.1118 & \\
a5 & 2 & 10 & 2 & 10 & 0.2257 & 0.6346 & \\
a6 & 8 & 40 & 3 & 14 & 3.4503 & 0.0632 & \\
a7 & 1 & 5 & 5 & 24 & 1.5908 & 0.2072 & \\
a8 & 0 & 0 & 1 & 5 & 0.0006 & 0.9802 & \\
a9 & 3 & 15 & 3 & 14 & 0.1423 & 0.7059 & \\
a10 & 6 & 30 & 7 & 33 & 0.0525 & 0.8186 & \\
a11 & 5 & 25 & 6 & 29 & 0.0665 & 0.7964 & \\
a12 & 0 & 0 & 1 & 5 & 0.0006 & 0.9802 & \\
a13 & 0 & 0 & 0 & 0 & & & \\
TNF-308*2 & 6 & 30 & 3 & 14 & 0.7017 & 0.4022 & \\
HLA-DRB1*03 & 6 & 30 & 4 & 19 & 0.2047 & 0.6509 & \\
HLA-DRB1*15 & 10 & 50 & 3 & 14 & 6.0341 & 0.0140 & 0.18 \\
HLA-DRB1*15 & 17 & 85 & 6 & 28.6 & 13.244 & 0.0002 & 0.0068 \\
and/or TNFa2 & & & & & & \\
\hline
\end{tabular}

PR: pulmonary responders; PNR: pulmonary nonresponders; Pc: Bonferroni correction.

Table 4.-Occurrence of tumour necrosis factor (TNF)a2 microsatellite in human leukocyte antigen (HLA)DRB1*15-positive and DRB1*15-negative subjects

\begin{tabular}{lcccc}
\hline Subject & $\mathrm{n}$ & HLA & $\mathrm{n}$ & TNFa2 (\%) \\
\hline PR & \multirow{2}{*}{20} & DRB1*15-pos & 10 & $6(60)$ \\
& & DRB1*15-neg & 10 & $7(70)$ \\
PNR & 21 & DRB1*15-pos & 3 & $1(33.3)$ \\
& & DRB1*15-neg & 18 & $3(16.6)$ \\
Control & 51 & DRB1*15-pos & 11 & $3(27.4)$ \\
& & DRB1*15-neg & 40 & $13(32.5)$ \\
\hline
\end{tabular}

PR: pulmonary responder; PNR: pulmonary nonresponder.

the polymorphic tumour necrosis factor-a2 microsatellite, reported to increase tumour necrosis factor production, supports a critical role of this cytokine in this disease. In addition, the present results also suggest that the genotyping for human leukocyte antigen-DRB1 and tumour necrosis factor polymorphisms might be useful for the planning of the chemotherapy.

Acknowledgements. The authors would like to thank A. Sanchez-Mazas for help with the statistical calculations, J. Aniol for help with the study of the chest radiography, and $\mathrm{R}$. Zanone for expert technical assistance.

\section{References}

1. Mcdonald JA. Idiopathic pulmonary fibrosis. A paradigm for lung injury and repair. Chest 1991; 99: $87 \mathrm{~s}-93 \mathrm{~s}$.
2. Thrall RS, Scalise PJ. Bleomycin. In: Phan SH, Thrall RS, eds. Pulmonary fibrosis. New York, Dekker, 1995; pp. 231-292.

3. Rossi GA, Szapiel S, Ferrans VJ, Crystral RG. Susceptibility to experimental interstitial lung disease is modified by immune and non immune-related genes. Am Rev Respir Dis 1987; 135: 448-455.

4. Piguet PF, Vesin C. Pulmonary platelet trapping induced by bleomycin: correlation with fibrosis and involvement of the beta 2 integrins. Inter J Exp Pathol 1994; 75: 321-328.

5. Piguet PF, Collart MA, Grau GE, Kapanci Y, Vassalli P. Tumor necrosis factor/cachectin plays a key role in bleomycin-induced pneumopathy and fibrosis. J Exp Med 1989; 170: 655-663.

6. Piguet PF, Vesin C. Treatment of bleomycin or silicainduced pulmonary fibrosis in mice by a human recombinant soluble TNF receptor. Eur Respir $J$ 1994; 7: 515-518.

7. Campbell RD, Trowsdale J. Map of the human MHC. Immunol Today 1993; 14: 349-352.

8. Browning JL, Miatkowski K, Sizing I, et al. Signaling through the lymphotoxin beta receptor induces the death of some adenocarcinoma tumor lines. $J$ Exp Med 1996; 183: 867-878.

9. Wilson AG, de Vries N, Pociot F, Di Giovine FS, van der Putte LBA, Duff GW. An allelic polymorphism within the human tumor necrosis factor alpha promoter region is strongly associated with HLA A1, B8 and DR3 alleles. J Exp Med 1993; 177: 557-560.

10. Wilson AG, Symons JA, McDowell TL, McDevitt HO, Duff GW. Effects of a polymorphism in the human tumor necrosis factor alpha promoter on transcriptional activation. Proc Natl Acad Sci USA 1997; 94: 3195-3199.

11. Moffatt MF, Cookson WOCM. Tumor necrosis factor haplotypes and asthma. Hum Mol Genet 1997; 6: 551-554.

12. McGuire W, Hill VS, Allsopp CEM, Greewood BM, Kwjatkowski D. Variation in the TNF-a promotor region associated with susceptibility to cerebral malaria. Nature 1994; 371: 508-511.

13. Pena AS, Garrote JA, Crusius JB. Advances in the immunogenetics of coeliac disease. Clues for understanding the pathogenesis and disease heterogeneity. Scand J Gastroenterol Suppl 1998; 225: 56-58.

14. Udalova IA, Nedospasov SA, Webb GC, Chaplin DD, Turetskaya RL. Highly informative typing of the human TNF-locus using six adjacent polymorphic markers. Genomics 1993; 16: 180-186.

15. Pociot F, Briant L, Jongeneel CV, et al. Association of tumor necrosis factor (TNF) and class II major histocompatibility complex alleles with the secretion of TNF-alpha and TNF-beta by human mononuclear cells: a possible link to insulin-dependent diabetes mellitus. Eur J Immunol 1993; 23: 224-231.

16. Cabrera M, Shaw MA, Sharples C, et al. Polymorphism in tumor necrosis factor genes associated with mucocutaneous leishmaniasis. J Exp Med 1995; 182: 1259-1264.

17. Marshall RP, McAnulty RJ, Laurent GJ. The pathogenesis of pulmonary fibrosis: is there a fibrosis gene? Int J Biochem Cell Biol 1997; 29: 107-120.

18. Briggs DC, Vaughan RW, Welsh KI, Myers A, Dubois RM, Black CM. Immunogenetic prediction of pulmonary fibrosis in systemic sclerosis. Lancet 1991; 338: 661-662. 
19. Zhai R, Jetten M, Schins RPF, Franssen H, Born PJA. Polymorphisms in the promoter of the tumor necrosis factor-alpha gene in coal miners. $A m ~ J$ Ind Med 1998; 34: 318-324.

20. Connors J, Klimo P, Adams G, et al. Treatment of advanced Hodgkin's disease with chemotherapycomparison of MOPP/ABV hybrid regimen with alternating courses of MOPP and ABVD: a report from the National Cancer Institute of Canada clinical trials group. J Clin Oncol 1997; 15: 1638-1645.

21. Pavy JJ, Denekamp J, Letschert J, et al. EORTC late effects working group. Late effects toxicity scoring: the SOMA scale. Radiother Oncol 1995; 35: 11-15.

22. Tiercy J, Grundschober C, Jeannet M, . A comprehensive HLA-DRB, DQB, and DPB oligotyping procedure by hybridization with sequence-specific oligonucleotide probes. In: Bidwell J, Hui K, eds. Handbook of HLA typing techniques. USA, CRC Press Inc, 1993; pp. 117-147.

23. Verjans G, Brinkman B, Van Doornik C, Kijlstra A, Verweij C. Polymorphism of tumor necrosis factoralpha (TNF-a) at position -308 in relation to ankylosing spondylitisl. Clin Exp Immunol 1994; 97: 45-47.

24. Ruuls SR, Sedgwick JD. Immunogenetics 99: unlinking tumor necrosis factor biology from the major histocompatibility complex: lessons from human genetics and animal models. Am J Hum Genet 1999; 65: 294-301.

25. Klitz W, Aldrich CL, Fildes N, Horning SJ, Begovich AB. Localization of predisposition to Hodgkin Disease in the HLA class II region. Am J Hum Genet 1994; 54: 497-505.

26. Whilborg C, Sjoberg J, Intaglietta M, Axdroph U, Pisa EK, Pisa P. Tumor necrosis factor-alpha cytokine promoter gene polymorphism in Hodgkin's disease and chronic lymphocytic leukaemia. Brit J Haematol 1999; 104: 346-349.

27. Gallagher G, Eskdale J, Oh HH, Richards SD, Campbell DA, Field M. Polymorphism in the TNF gene cluster and MHC serotypes in the west Scotland. Immunogenetics 1997; 45: 188-194.

28. Jacob CO, Fronek Z, Lewis GD, Koo M, Hansen JA, McDevitt HO. Heritable major histocompatibility complex class-II-associated differences in production of tumor necrosis factor-alpha-relevance to genetic predisposition to systemic lupus erythematosus. Proc Natl Acad Sci USA 1990; 87: 1233-1237.

29. Turner D, Grant SCD, Lamb WR, et al. A genetic marker of high TNF-alpha production in heart transplant recipients. Transpl 1995; 60: 1113-1117. 